\title{
Review
}

\section{From Prodromal to Overt Parkinson's Disease: Towards a New Definition in the Year 2040}

\author{
Daniela Berg ${ }^{\mathrm{a}, \mathrm{b}, *}$ and Ronald B. Postuma ${ }^{\mathrm{c}, *}$ \\ ${ }^{a}$ Department of Neurology, Christian-Albrechts-University of Kiel, Kiel, Germany \\ ${ }^{\mathrm{b}}$ Department of Neurodegeneration, Hertie-Institute for Clinical Brain Research Tuebingen, Germany \\ ${ }^{\mathrm{c}}$ Department of Neurology, Montreal General Hospital, Montreal, Quebec, Canada
}

Accepted 11 November 2018

\begin{abstract}
The field of prodromal PD is still in its infancy, and at the cusp of major advances. This article summarizes where we are, and most importantly where we need to go in order for the promise of prodromal PD to be realized. In the immediate future, the criteria need to be updated with additional markers and disseminated broadly. In the near future, they need to better incorporate changes in likelihood ratio with age and sex, combine markers in novel ways using big data approaches, identify subtypes, and incorporate better higher-specificity markers as they are discovered. Integration of smartphone/wearable markers and biomarkers of progression from the prodromal phase will allow development of neuroprotective trials in early stages. By 2040, it is hoped that prodromal criteria will be incorporated into active neuroprotective treatment programs, allowing a program of population-based screening followed by early treatment and ultimately the prevention of clinical PD from ever becoming manifest.
\end{abstract}

Keywords: Prodromal Parkinson's disease, higher-specificity markers, big data approaches, progression markers, subtypes, population-based screening, neuroprotective trials, gene-specific therapy, individual mechanism-specific therapy

\section{WHAT WE ACHIEVED}

In the last 25 years our understanding of the evolution of Parkinson's disease (PD) has changed dramatically. Brilliant work of many in different scientific fields paved the way for the concept of prodromal PD; that is, a phase of years to decades in which non-motor and subtle motor symptoms may indicate spreading PD pathology, but do not meet the threshold for diagnosis according to the classic motor-based clinical criteria [1].

The main anchors of this concept are:

${ }^{*}$ Correspondence to: Daniela Berg, MD, Department of Neurology, Christian-Albrechts-University of Kiel, Arnold-Heller-Str. 324105 Kiel, Germany. E-mail: Daniela.berg@uksh.de. and Ronald B. Postuma, MD, MSc, Department of Neurology, L7-305 Montreal General Hospital, 1650 Cedar Ave, Montreal, Canada H3G1A4. E-mail: ron.postuma@mcgill.ca.
- the broadly accepted fact that the neurodegenerative process in PD is a slowly spreading process, possibly starting in the gut or olfactory system $[2,3]$ and finally encompassing much of the nervous system;

- increasing knowledge of risk factors and clinical symptoms that antecede the typical motor manifestations by years to decades, and can be correlated to imaging and histopathological findings;

- longitudinal studies that observed conversion to PD in cohorts of individuals with different combinations of risk and prodromal markers

These advances allowed the establishment of a mathematical model based on Bayesian statistics that enables one to calculate an individual's personal risk of being in the prodromal phase of PD [4]. 


\section{LIMITATIONS AND SHORTCOMINGS OF THE CURRENT UNDERSTANDING AND MODEL}

The prodromal PD criteria are meant to be research criteria, and constitute a first step in what should be a continually-updated process.

Several shortcomings have been noted, both at the time of publication and as several groups have studied the criteria. These include:

1. Prodromal state vs. time to conversion: As stated in the original criteria the statistical model can only give the likelihood that an individual is in the prodromal phase - whether and when classical motor symptoms will develop can currently not be predicted by this model. Further information is therefore required to predict time of phenoconversion.

2. Independency of markers: Most prospective studies used in establishing the criteria tested single markers; however, criteria will in general be applied to individuals most likely carrying several markers. If markers are not independent of each other, the model breaks down. Fortunately, it seems that although many non-motor symptoms that precede motor PD are also common in the non-PD elderly population, their combination is quite rare in individuals without PD [5]. Early tests have suggested that markers are indeed independent, and so can be combined $[6,7]$.

3. Age/sex effects: Currently, age and sex are incorporated into risk estimates, but not in the LR of markers themselves. However, the LR of many markers seems to vary with age and sex; this needs to be incorporated in the criteria [8].

4. Subtypes with undetectable prodromal stages: PD is characterized by a broad phenotypic spectrum and so is prodromal PD. Some PD patients have very few non-motor symptoms; so, these individuals will have hardly any of the nonmotor markers that are required for diagnosis of the prodromal phase [9].

5. Continuous vs. dichotomous cutoffs: The criteria use dichotomous cutoffs; however, certain markers are measurable as continuous variables, which could potentially provide different LR at different cutoffs. Moreover, some studies indicate markers which can vary and even revert to normal, like olfactory dysfunction [10]; therefore, it should not be forgotten that continual re-evaluation of markers is required.

6. Limited markers: Many studies have found that criteria were relatively insensitive. This reflects the reality of the field of PD prediction; so far, there are only a few markers that are sufficiently powerful to identify high LR of PD. However, already since publication of the current criteria for prodromal PD [4] several markers have been found that could be added to the prediction model. Examples include diabetes, physical inactivity, and low plasma urate levels in men. As the field evolves, more will be discovered, and sensitivity will continue to improve.

7. So far the prodromal criteria are composed of clinical and (few) imaging signs. Although promising approaches in biomarker research can be noted no biofluid or histological marker has proven sufficiently sensitive and specific to be included in the criteria.

This is even more significant as the current prodromal criteria are primarily based on clinical signs with no opportunity to include brain autopsy as ultimate diagnostic confirmation.

\section{WHAT IS CURRENTLY BEING DONE}

To better understand and constantly update the criteria, an internet platform, free to the scientific community, is currently being designed which will updated LRs, provide a calculator that can be used to calculate probability for individual patients, and a forum for scientific exchange among experts to further refine the criteria.

Moreover, the ongoing extensive research of epidemiologists, basic scientists, clinicians and neuroimaging specialists will lead to the detection of new risk factors and biomarkers and will substantiate, expand and deepen our understanding of the very early phases of PD. Combined approaches that test multiple modalities in the same patient are especially promising. These include multimodal imaging (e.g., to stage REM sleep behavior disorder [11]) as well as multimodal biomarker platforms, incorporating combinations of biomarkers from different sources.

Now, "big data" analyses of huge data sets have also entered PD research. Especially the fields of genetics (see for example [12], which combines data from $>8$ million individuals), -omics approaches and the continuous capturing of movements in daily life 
by various wearables and devices, including smartphones (e.g., [13]. These will benefit from new methods of data handling and analyses.

Importantly, it needs to be appreciated that merging of large sets of data has become possible not only due to secure web applications (e.g., Redcap https://www.project-redcap.org/) but - and primarily due to - a scientific spirit of cooperation and data sharing.

\section{EXPECTED FINDINGS: THE NEAR FUTURE}

Use of the anticipated internet platform for prodromal PD will accelerate learning and application of the criteria in a growing community of scientists-expanding its use not only to cohorts with the endpoint of PD/neurodegeneration but also to different primary endpoints in which additional markers may be identified. This should help facilitate detection and dissemination of currently unknown risk and prodromal markers for PD which include besides clinical and neuroimaging also biofluid, histological, etc., markers.

Additionally, an important further kind of marker will become deducible from prodromal longitudinal cohort studies, namely prodromal progression markers. These will be essential as endpoints for studies which aim to slow disease progression in the prodromal stages.

With new data arising from objective movement measurements, the earlier detection of motor symptoms will become possible. Objectively-measured markers such as reduced unilateral arm swing [14], impaired balance $[15,16]$, wearable (e.g., mobilephone) based markers of activity etc. indicate that we can expect to change our understanding of early motor PD. Keeping in mind that still several issues regarding sensor based assessments need to be solved including compliance and potential selection bias in populations who are willing to wear devices, a paradigm shift regarding appearance of first motor abnormalities and relationship to the current cardinal motor symptoms is likely.

\section{CHALLENGES FOR THE INTERMEDIATE FUTURE}

PD is currently still defined by its characteristic motor symptoms and we do not know, whether the underlying neurodegeneration starting outside the brain will inevitably progress to the brain in all individuals, neither do we know the individual pace of progression. The questions are: When is the starting point of PD? What will define the disease; will it still be motor symptoms (possibly typical subtle ones), or will it be biomarker evidence of nigrostriatal system neurodegeneration without motor symptoms? Will it be a certain combination of non-motor signs? Or will it be based upon non-clinical biomarkers, similar to changes in Alzheimer's disease?

Given the fact that some individuals with Parkinson's disease suffer more from non-motor symptoms [17] than motor ones in the early stages, our aim will need to focus not only on stopping motor progression but also those manifestations outside the substantia nigra.

Understanding prodromal PD will mean understanding PD in all its heterogeneity. Therefore subtypes need to be considered and accounted for in the predictive models.

Finally, progress needs to continue on mitigating all the limitations noted above.

\section{WHAT IS NEEDED TO MEET THESE CHALLENGES?}

Progress of the recent years was only possible due to sincere and open-minded collaboration of many scientists in different fields. We will need to further combine forces. Cohort studies will need to cross epidemiological, clinical and basic science boundaries, so that we move beyond clinical markers of prodromal disease. Harmonization of assessments, an integrative attitude towards other disciplines, and deeper data sharing and analyses will be essential.

Last, but definitely not least, the current criteria are established as research criteria and have been mainly disseminated to specialists in the field. However, advances in this field are not hidden in our information society. As the general public begins to grasp our evolving understanding of early PD stages, new challenges will emerge related to potential for harm. Identifying oneself or ones loved one as being in prodromal stages of neurodegeneration, without any preventative treatment can lead to excessive distress, overplanning (e.g., retiring long before disease onset), use of unproven preventative therapies, discrimination at work, inability to obtain insurance, etc. Until preventative therapy is developed, we must become increasingly prepared to help those directly affected by our research findings. 


\section{THE VIEW FROM 2040: A FUTURE WITHOUT CLINICAL PD?}

As we better understand the prodromal phase and increase diagnostic certainty, we will inevitably begin to understand that our current concept of prodromal PD is, in fact, PD. In 2040 we also will have gained understanding of progression in the prodromal phase, and the differing prodromal evolution of subtypes of PD. As subtype-specific biomarkers are understood, diagnostic accuracy will continue to improve.

The primary goal of all prodromal research is help slow or stop disease in its early stages, and prevent clinical disease onset. Ultimately, one would hope to prevent initiation of the neurodegenerative process at all.

For our research to bear fruit, we also require a better understanding of causative mechanisms, to enable the discovery of true disease modifying and finally neuroprotective treatments.

Imagine that the search for prodromal bio- and progression markers has become successful, so that neurodegenerative diseases can be detected and prevented. What might this entail for society? A possible outcome is a future in which every member of the population, starting in early midlife undergoes periodic complete evaluations for all neurodegenerative diseases. This could be universal, or if knowledge about genetic risk factors advances sufficiently, could be a personalized biomarker screening based upon genetic risk load and one's individual genetic pathways that contribute to neurodegeneration. Screening might start with easy to obtain functional clinical measures, or blood- and imaging-biomarkers to identify the earliest stages of neurodegeneration. Depending on the specificity of the early tests, further assessments like multimodal PET scanning for protein deposition may be added.

If early disease is detected, a course of treatment is started. This will again be personalized, based on the genetic pathways contributing to the neurodegenerative process identified, perhaps including changes in lifestyle (e.g., influencing the microbiome or enhancing compensatory brain mechanisms by individual training, etc.), gene-specific therapy, individual mechanism-specific therapy (antioxidants, mitochondria support, liposomal enhancement), periodic protein-based clearing (e.g., a 6-months course of amyloid-reducing therapy each decade), or a well-tolerated small molecule protein inhibitor given lifelong.
As daunting or unobtainable as this might seem, such a scaled-up prevention of pathological brain aging will allow society to catch up to its advances in disease prevention outside the brain, leading to ever longer and healthier lives without inevitable neurologic disability. A game-changing outcome like this needs to be our goal for 2040 .

\section{CONFLICTS OF INTEREST}

The authors have no conflict of interest to report regarding this manuscript.

\section{REFERENCES}

[1] Postuma RB, Berg D, Stern M, Poewe W, Olanow CW, Oertel W, Obeso J, Marek K, Litvan I, Lang AE, Halliday G, Goetz CG, Gasser T, Dubois B, Chan P, Bloem BR, Adler $\mathrm{CH}$, Deuschl G (2015) MDS clinical diagnostic criteria for Parkinson's disease. Mov Disord 30, 1591-1601.

[2] Braak H, Del Tredici K, Bratzke H, Hamm-Clement J, Sandmann-Keil D, Rüb U (2002) Staging of the intracerebral inclusion body pathology associated with idiopathic Parkinson's disease (preclinical and clinical stages). J Neurol 249(Suppl 3), III/1-5.

[3] Borghammer P (2018) How does Parkinson's disease begin? Perspectives on neuroanatomical pathways, prions, and histology. Mov Disord 33, 48-57.

[4] Berg D, Postuma RB, Adler CH, Bloem BR, Chan P, Dubois B, Gasser T, Goetz CG, Halliday G, Joseph L, Lang AE, Liepelt-Scarfone I, Litvan I, Marek K, Obeso J, Oertel W, Olanow CW, Poewe W, Stern M, Deuschl G (2015) MDS research criteria for prodromal Parkinson's disease. Mov Disord 30, 1600-1611.

[5] Hughes K, Gao X, Baker J, Schwarzschild M, Ascherio A (2017) Associations of probable REM sleep behavior disorder, constipation, and hyposmia with PD. In: Movement Disorder Society: Proceedings of the International Congress of Parkinson's Disease and Movement Disorders. Wiley, Vancouver, BC, Canada.

[6] Fereshtehnejad S-M, Montplaisir JY, Pelletier A, Gagnon J-F, Berg D, Postuma RB (2017) Validation of the MDS research criteria for prodromal Parkinson's disease: Longitudinal assessment in a REM sleep behavior disorder (RBD) cohort. Mov Disord 32, 865-873.

[7] Chen H, Huang X, Guo X, Peddada S (2014) Individual and joint prevalence of three nonmotor symptoms of PD in the US general population. Mov Disord 29, 1316-1319.

[8] Heinzel S, Kasten M, Behnke S, Vollstedt E-J, Klein C, Hagenah J, Pausch C, Heilmann R, Brockmann K, Suenkel U, Yilmaz R, Liepelt-Scarfone I, Walter U6, Berg D (2018) Age- and sex-related heterogeneity in prodromal Parkinson's disease. Mov Disord 33, 1025-1027.

[9] Pilotto A, Heinzel S, Suenkel U, Lerche S, Brockmann K, Roeben B, Schaeffer E, Wurster I, Yilmaz R, LiepeltScarfone I, von Thaler AK, Metzger FG, Eschweiler GW, Postuma RB, Maetzler W, Berg D (2017) Application of the movement disorder society prodromal Parkinson's disease research criteria in 2 independent prospective cohorts. Mov Disord 32, 1025-1034. 
[10] Heinzel S, Roeben B, Ben-Shlomo Y, Lerche S, Alves G, Barone P, Behnke S, Berendse HW, Bloem BR, Burn D, Dodel R, Grosset DG, Hu M, Kasten M, Krüger R, Moccia M, Mollenhauer B, Oertel W, Suenkel U, Walter U, Wirdefeldt K, Liepelt-Scarfone I, Maetzler W, Berg D (2016) Prodromal markers in Parkinson's disease: Limitations in longitudinal studies and lessons learned. Front Aging Neurosci $\mathbf{8}, 147$.

[11] Knudsen K, Fedorova TD, Hansen AK, Sommerauer M, Otto M, Svendsen KB, Nahimi A, Stokholm MG, Pavese N, Beier CP, Brooks DJ, Borghammer P (2018) In-vivo staging of pathology in REM sleep behaviour disorder: A multimodality imaging case-control study. Lancet Neurol 17, 618-628.

[12] De Pablo-Fernandez E, Goldacre R, Pakpoor J, Noyce AJ, Warner TT (2018) Association between diabetes and subsequent Parkinson disease: A record-linkage cohort study. Neurology 91, e139-e142.

[13] Prince J, Arora S, de Vos M (2018) Big data in Parkinson's disease: Using smartphones to remotely detect longitudinal disease phenotypes. Physiol Meas 39, 44005.
[14] Mirelman A, Bernad-Elazari H, Thaler A, Giladi-Yacobi E, Gurevich T, Gana-Weisz M, Saunders-Pullman R, Raymond D, Doan N, Bressman SB, Marder KS, Alcalay RN, Rao AK, Berg D, Brockmann K, Aasly J, Waro BJ, Tolosa E, Vilas D, Pont-Sunyer C, Orr-Urtreger A, Hausdorff JM, Giladi N (2016) Arm swing as a potential new prodromal marker of Parkinson's disease. Mov Disord 31, 1527-1534.

[15] Schrag A, Horsfall L, Walters K, Noyce A, Petersen I (2015) Prediagnostic presentations of Parkinson's disease in primary care: A case-control study. Lancet Neurol 14, 57-64.

[16] Maetzler W, Mancini M, Liepelt-Scarfone I, Müller K, Becker C, van Lummel RC, Ainsworth E, Hobert M, Streffer J, Berg D, Chiari L (2012) Impaired trunk stability in individuals at high risk for Parkinson's disease. PLoS One 7, e32240.

[17] Schapira AHV, Chaudhuri KR, Jenner P (2017) Non-motor features of Parkinson disease. Nat Rev Neurosci 18, 509. 